\title{
Sobre inundaciones y anegamientos
}

Flood Disasters

Francisco J. Ferrando A.

\section{Filiación}

Académico del D. de geografía de la F.A.U. de la Universidad de Chile fferrand@uchilefau.cl

\section{Resumen}

Respecto a anegamientos e inundaciones, el autor realiza algunas precisiones conceptuales que afectan la gestión de acciones preventivas, la planificación y el ordenamiento territorial; además se ofrece una sistematización del quehacer sobre las inundaciones.

Palabras clave

Manejo de cuencas, aguas lluvia, inundaciones, desastres naturales (manejo, prevención).

\section{Abstract}

The author punctualizes the concepts regarding preventive actions and territorial planning. Also the article includes a systematized list of actions related to flood management.

\section{Key words}

Flood management, rain drainage, watershed management, floods, natural disasters.

\section{Sumario}

Introducción

Características de las inundaciones

Los anegamientos

Situaciones mixtas: inundación + anegamiento

Escala de los eventos y posibilidades de gestión

Aportes del manejo de cuencas

El tema de la alerta temprana

Sistematizando el quehacer sobre las inundaciones

Conclusiones: tropezando con la misma piedra

Bibliografía

Otras fuentes 


\section{Introducción}

Considerando los acontecimientos registrados en parte importante del país relacionados con eventos pluviométricos de Julio del 2006, así como en múltiples otros inviernos, parece pertinente establecer algunas precisiones y diferenciaciones respecto de esta temática, tanto conceptuales como de sus derivaciones en el terreno de la gestión y de la factibilidad de acciones preventivas.

Es común escuchar que se habla de inundaciones y de anegamientos como si fueran sinónimos. Si bien ello puede parecer aceptable en el lenguaje vernacular, no lo es en términos de la implicancia que ello tiene a nivel de la planificación y el ordenamiento territorial. En términos simples, una inundación corresponde a una masa de agua que se sale de su cauce y se dispone temporalmente fuera de él. Diferente es un anegamiento, el cual se define como la acumulación temporal de aguas lluvias en una determinada porción de territorio.

Aclarada esta diferencia, sobre la cual se ahonda más adelante, es claro que las relaciones causales también lo son. Siendo así, también lo serán las posibles acciones de mitigación, elusión, control, etc. Por lo tanto, esta diferenciación tiene gran importancia cuando se enfoca esta problemática desde el punto de vista de la gestión preventiva ante ambas situaciones, así como de los problemas que de ellas se derivan.

\section{Características de las inundaciones}

Las inundaciones propiamente tales corresponden a una consecuencia derivada de otros procesos de recurrencia interanual, como son las crecidas de los cursos de agua, sumado ello a condiciones de insuficiencia de los sistemas de evacuación, sean estos cauces naturales, sistemas de drenaje artificializados, colectores urbanos, etc.

Se trata del resultado del desequilibrio que se manifiesta en un momento, lugar y situación dada, entre el volumen hídrico a evacuar en una determinada parcela de tiempo, y la capacidad de evacuación de los cauces o sistemas de drenaje o, en otras palabras, la oferta de cauce se ve superada por la demanda de cauce. Debe tenerse en cuenta, además, que dicha demanda no está compuesta sólo por agua, sino también por los sedimentos que esta transporta y arrastra, y cuya proporción respecto del volumen hídrico, sumado a las variaciones en la capacidad de carga del curso de agua, va a influir directamente en la ocurrencia de los desbordes.

Un hecho relevante es la recurrencia de las crecidas que presenta una cuenca fluvial dada respecto de otra. Ello está asociado, por una parte, a las características del régimen pluviométrico y térmico que registre el clima imperante $y$, por otra, a las características morfométricas que está presente (alturas, forma, pendiente media, superficie, etc.), al desarrollo del sistema de drenaje (densidad, frecuencia y jerarquía de la red hídrica), y a la capacidad de retención hídrica de la cuenca, aspectos todos ellos que influyen en la torrencialidad, la velocidad de respuesta, el tiempo de concentración, y el volumen de los caudales.

Respecto de la carga sedimentaria y su relación con los desbordes, esto tiene como explicación el hecho que las variaciones de pendiente en el eje longitudinal de los cauces provocan modificaciones en la velocidad con que escurre el agua, lo que motiva la sedimentación o abandono local de la carga de sedimentos provocando 
consecuentemente una reducción de la sección transversal $y$, por lo tanto, de la capacidad de estos cauces para contener y evacuar las aguas.

En otros casos, cuando se trata de inundaciones o "salidas de madre" en condiciones de caudales "normales" (altas aguas medias), la causa suele encontrarse en la reducción de la sección de los colectores en forma artificial (angostamiento por urbanización, relleno por desechos, etc.), natural (sedimentación progresiva), o accidental (eventos que provocan obstrucción parcial o total) como son los deslizamientos de tierra, los derrumbes, la caída de árboles, el derrumbe de puentes, etc.

\section{Los anegamientos}

Habiendo sido definidos como formas de acumulación de aguas de lluvia sobre el terreno, y oteando hacia la causalidad, por lo general en este caso también existe una situación de desbalance, la cual se da entre el volumen de la lluvia precipitada en un determinado lapso de tiempo y la capacidad de evacuación de un suelo dado, tanto horizontal como verticalmente.

Si bien ello es cierto, existen complicaciones. Estas se derivan de los cambios en la permeabilidad, en la saturación del suelo y en la micro-topografía de la superficie, ya sean por causas naturales o artificiales.

Los cambios en la permeabilidad del suelo pueden ser positivos o negativos. Entre las acciones positivas, es decir, que incrementan la permeabilidad está la aradura y la incorporación de materia orgánica. Los efectos negativos tienen como causa la intervención en términos de compactación $\mathrm{y} / \mathrm{o}$ de cobertura con materiales impermeables, como son las construcciones, los concretos y los asfaltos, incluso la aplicación de petróleo o aceite quemado a los caminos de tierra o la adición de una cubierta de maicillo y su apisonamiento.

La compactación por su parte, no sólo tiene relación con el uso de maquinaria pesada en caminos y terrenos a ser edificados, sino también con la conformación de rellenos mediante escombros y tierras arcillosas sobre suelos permeables (por Ej.: generación de suelos industriales sobre el humedal de Batuco), y con el cambio de los bosques nativos por especies forestales, ya que esto implica el paso de formaciones con tres estratos vegetacionales (arbóreo, arbustivo y herbáceo) a plantaciones tipo parque, sólo con el estrato arbóreo, lo que redunda en una menor capacidad de retención e infiltración de los terrenos.

La saturación del suelo se refiere concretamente a dos situaciones: $1^{\circ}$ - la existencia natural de áreas hidromórficas o con presencia semi-constante a constante de la napa en superficie, lo que impide la percolación de las aguas lluvias, puesto que un suelo con estas características se comporta como un material impermeable $y, 2^{\circ}$ - los suelos pueden sufrir saturación progresiva, más o menos rápida según su permeabilidad, ante la ocurrencia de precipitaciones prolongadas o intensas, lo cual conduce a una situación similar a la anterior. Se trata del concepto de permeabilidad efectiva.

En este caso, si un suelo dado ve superada su capacidad de infiltración y evacuación subterránea de las aguas, lo cual es común que ocurra cuando las lluvias son intensas, 
se generará un excedente pluviométrico que deberá permanecer en superficie por el lapso de tiempo post-Iluvia necesario para que la situación se normalice.

Las variaciones en la micro-topografía se refieren a cambios en la configuración del terreno que crean desniveles y obstáculos para el drenaje superficial. Este hecho tiene una causal fundamental: el hombre. Las construcciones que este efectúa (diques, terraplenes, camellones, soleras, muros, etc.) vienen a constituirse en obstáculos para que el agua circule libremente sobre los terrenos siguiendo las diferencias de pendiente. Esto ocurre tanto en el ámbito rural como en el urbano.

En el primero de los casos, los terraplenes de las carreteras y de las vías férreas suelen cumplir el rol de diques por insuficiencia de alcantarillas transversales, generando enlagunamientos hacia aguas arriba. En las ciudades, prácticamente todas las construcciones son obstáculos al escurrimiento de las aguas lluvias, las cuales terminan por acumularse en sectores ligeramente más bajos sin posibilidad de circular ni infiltrarse.

Frente a la ocurrencia de precipitaciones, la malla de calles y avenidas de una ciudad se transforma en una "red fluvio-vial" que, siguiendo la inclinación del eje de cada una de ellas funcionando como cauces, va creando flujos que confluyen progresivamente, sumando sus aguas en dirección de los sectores topográficamente más deprimidos. Creo que con base en un levantamiento topográfico detallado de las calles de una ciudad, es posible establecer claramente de donde vienen y para donde van las aguas, y los diferentes puntos de concentración, lo cual puede servir como herramienta de decisión sobre qué hacer y donde hacer en el contexto de las medidas que deben emanar de los planes maestros de aguas lluvias.

Por otra parte, cuando los anegamientos en el área rural llegan a superar parte de los obstáculos del terreno, estas aguas comienzan a desplazarse sobre la superficie, pasando a constituir una forma de escurrimiento laminar. Como resultado de este proceso pueden presentarse nuevas situaciones:

- Pueden resultar afectados terrenos que no habían sufrido este problema;

- La suma de estas aguas provenientes de diferentes sectores, lo cual requiere de ciertas condiciones topográficas favorables, puede elevar sustantivamente la cota de anegamiento en algún sector agravando la situación;

- En su camino pueden encontrarse con un cauce y vaciarse en él, lo cual puede ser la solución para un sector, pero puede provocar problemas aguas abajo al generar un superávit hídrico en dicho cauce y su desborde.

\section{Situaciones mixtas: inundación + anegamiento}

Suele suceder con frecuencia que ambos procesos se manifiesten conjuntamente en un determinado territorio, escenario en el cual las consecuencias serán una suma de los efectos $y$, por cierto, el impacto socioeconómico concluirá en un desastre de envergadura mayor. Es claro que si un cierto espacio geográfico cruzado por ríos y canales se encuentra anegado producto de las lluvias registradas $y$, paralela 0 seguidamente con ello se produce un desmadre de los cursos de agua, el espesor que alcanzará la lámina de agua sobre el suelo será mucho mayor, hecho que se verá 
acompañado por un aumento en la velocidad de la masa hídrica, llevando ello a un incremento del poder destructivo.

Evidentemente, se trata de situaciones complejas con escasa a nula posibilidad de manejo o mitigación durante su desarrollo, incluso a nivel de rescate de personas. Es aquí cuando por enésima vez se vuelve a plantear la necesidad de la prevención, de las intervenciones bien pensadas y reguladas en el medio natural, del adecuado dimensionamiento y diseño de las obras de infraestructura, de la necesidad de obras de drenaje acordes a la expansión urbana, etc., etc.

Lamentablemente, el despertar de estas necesidades que surge durante el desastre, se olvida rápidamente a los pocos días de transcurrido este. Las ideas de proyectos vuelven a irse a pique hasta la próxima inundación y/o anegamiento, momento durante la cual son reflotadas otra vez para, poco después iniciar un nuevo período de hibernación. Es una suerte de "ciclo hidro-ilógico" en que se vuelve reiteradamente a chocar con la misma agua. (Ver Fig.1).

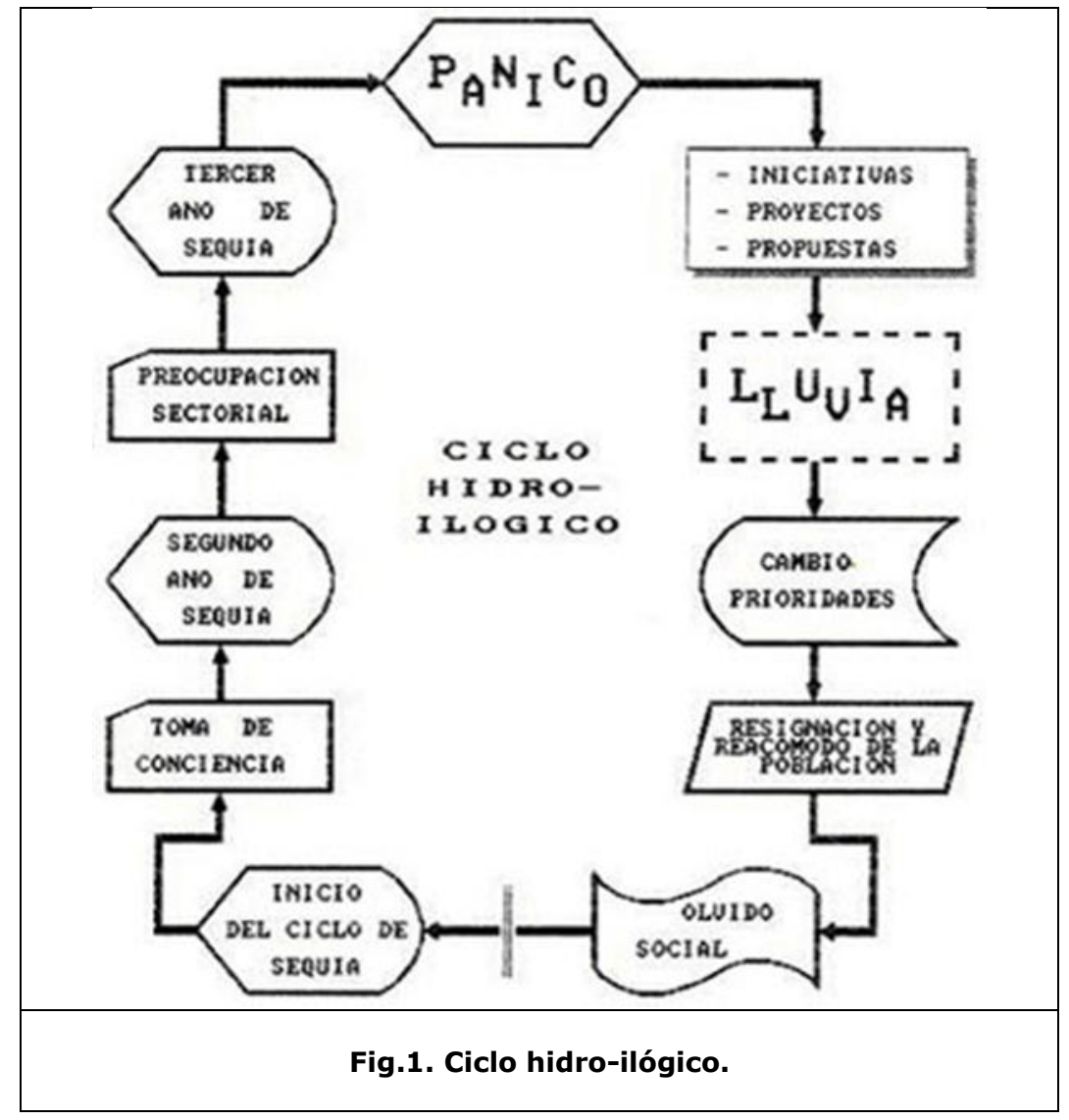

Se debe recordar además que, de acuerdo con este ciclo, las áreas que se han inundado históricamente son las que se siguen inundando. Ello sólo podría cambiar hacia el lado positivo (reducción de las áreas que se inundan) si hubieran cambios significativos en las relaciones causales previamente señaladas, pero ello requiere de 
múltiples acciones humanas específicas en cada una de las condicionantes naturales y antrópicas que propician, que colaboran en la ocurrencia de estos eventos. Pero, con los pies bien en la tierra (seca), también debemos reconocer limitaciones en este campo.

\section{Escala de los eventos y posibilidades de gestión}

En escala de magnitudes, debemos estar ciertos que existen eventos naturales exógenos, detonantes de inundaciones y anegamientos, muy diferentes. Ciertos eventos pluviométricos con carácter de diluvio, en que precipitan 150 o más milímetros en unas pocas horas y en una amplia extensión de territorio, es evidentemente una situación poco usual ante la cual cualquier medida o plan de prevención será insuficiente. Construir defensas para que no se desborde un río dado en su crecida centenaria está fuera de consideración y factibilidad. Con ello se quiere decir que las posibilidades de intervención preventiva están dimensionadas y pueden ser efectivas para mitigar, para ejercer un cierto control, sobre aquellos eventos que tienen una recurrencia de 10 a 15 años. En el primer caso, se trasciende a una situación de riesgo asumido (evento no controlable), frente a lo que los paneles internacionales suelen recomendar la evacuación previa y el preparar la ayuda necesaria para el resarcimiento de las pérdidas ocasionadas.

En el caso de inundaciones producto del desborde de cursos de agua por crecidas de recurrencia decenal o algo más, la prevención es factible no porque se pueda intervenir sobre el agente causal (por ejemplo: lluvias desmesuradas, o penetración de frentes cálidos que provocan lluvia sobre mantos nivales generando fusiones violentas), sino porque es posible hacerlo sobre los elementos del medio natural a nivel de superficie que reciben esta masa de agua y que deben responden a este in-put. Es allí, a este nivel, donde es posible entrometerse para mitigar las fuerzas de la madre naturaleza. Se trata de medidas estructurales y biológicas que buscan regular la respuesta de los cursos de agua de una hoya hidrográfica para, desde su nacimiento, colocar riendas a la manada hidrológica para que no se desboque cerro abajo. Ellas forman parte de las técnicas del "manejo de cuencas hidrográficas".

Respecto de los anegamientos, la situación es otra y, como se ha planteado, tiene dos caras. La cara rural del problema en que la mantención de la permeabilidad natural del suelo, y su mejora si es posible, pueden ayudar a una más rápida infiltración. También se pueden plantear obras como pozos de infiltración o canales de avenamiento, pero se trata de obras caras y cuyo efecto frente a lluvias intensas puede resultar claramente insuficiente. En estos casos, lo más efectivo es inobjetablemente el asentamiento de la población rural, casas y edificaciones complementarias, en sectores 2 a 3 metros por sobre la cota más baja del terreno en varias hectáreas a la redonda. El diseño de las casas debiera considerar, además, terraplenes sólidos y variables hidrodinámicas en su diseño, de modo de ofrecer la menor resistencia a la corriente, cuyo sentido de circulación suele ser el mismo anegamiento tras anegamiento.

En el rostro urbano de estos procesos, se repite el esquema general de confrontación entre un sistema socioeconómico nacional que funciona preferentemente en dirección Norte-Sur, y el sistema de procesos hidrológicos que funciona de Este a Oeste. Dentro del casco urbano las aguas procuran seguir dicho modelo, pero se ven entorpecidas y 
frenadas por una serie de obstáculos transversos, desde edificios a cunetas. Debe recordarse además que, en el caso de Santiago, la ciudad en su expansión ha borrado del modelado original una serie de cauces menores que daban salida a las aguas de la microcuencas precordilleranas, y que ha impermeabilizado miles de hectáreas en las que se producía infiltración.

En un escenario ficticio, es decir, haciendo abstracción de los sistemas de drenaje urbano existentes, se puede señalar que las aguas que pasan por una ciudad dada son la suma de dos totales: El total de las aguas lluvias y el total de las aguas de los esteros que drenan cuencas que nacen aguas arriba de la urbe. En un escenario real, las obras de avenamiento colaboran en parte, pero cada vez más insuficientemente por la velocidad de la expansión urbana, en absorber dicho volumen de agua. Dicha insuficiencia se hace patente cada vez que se registran precipitaciones en los sectores urbanos y su entorno.

Respecto de cursos de agua naturales o artificializados que circulan por las ciudades y sus costados, el estado de las secciones de las cuencas aguas arriba de estos centros es de vital trascendencia. Una cuenca sobreexplotada o mal manejada en su sección superior, o zona de producción del flujo hídrico y los sedimentos por ellos arrastrados, pierde de capacidad de retención y amortiguación de las aguas lluvias, se reduce su tiempo de respuesta, se infiltra menos agua y se generan torrentes difíciles de controlar, capaces de superar las dimensiones de las obras de control o protección, y capacidades de las de avenamiento.

Parece que antes las cosas se hacían mejor: El casco antiguo de la ciudad de Santiago por ejemplo no sufre anegamiento de sus calles. El resto de la ciudad cuando llueve es un laberinto vial de torrentes, esteros y lagunas. Se podría afirmar que la capacidad de las ciudades para absorber los efectos de eventos pluviométricos cambia en forma inversamente proporcional al ritmo de crecimiento areal de ellas. Esto se puede aplicar también a los sistemas de drenaje de aguas lluvias que deben formar parte de cada nueva urbanización. Son cada vez menos eficaces o simplemente trasladan el problema hacia otro sector urbano. La solución para el drenaje de las aguas lluvias aceptada por algunas Direcciones de Obras Municipales en el caso de condominios o poblaciones cerradas suele ser un agujero en el muro posterior, por el cual se arroja el agua hacia una población ubicada más abajo.

Con estos predicamentos, solucionar el problema de los anegamientos urbanos se ve muy lejano. Se han hecho algunos esfuerzos pero se necesita muchos más. Una medida de rápida ejecución es la no autorización de nuevas urbanizaciones en sectores bajos o micro-depresiones, en lugares donde el drenaje natural se presenta impedido, y en donde el nivel freático permanece o alcanza la superficie con frecuencia, principalmente en invierno.

Cada Plan Regulador debiera consignar estas áreas e indicar las restricciones pertinentes. El uso seguro del territorio debe ser determinado con criterios científicos y técnicos y no con argumentos economicistas. Se debe tener claridad que invertir en prevención no es un gasto, es un ahorro. Son los desastres que no ocurrirán y las pérdidas que no habrá que lamentar. 
De más largo plazo, corregir las insuficiencias en términos de medidas que ayuden a recoger y regular el paso de las aguas por las ciudades es absolutamente necesario. Algo se ha hecho al respecto. En Santiago, por ejemplo, existen poco más de 40 pozos de absorción de aguas lluvias, pero llama la atención que habiendo sido construidos por el organismo público pertinente, su ubicación no coincida totalmente con los 11 sectores que el mismo organismo reconoce como con riesgo por inundación. Es más, tampoco se aprecia relación con la información del MINVU de 1998 sobre los sectores inundados, información que fue recogida en la carta de riesgos por inundación desarrollada en el seno del Proyecto OTAS para el GORE metropolitano de Santiago. (Ver Figs. 2, 3, 4 y 5.)

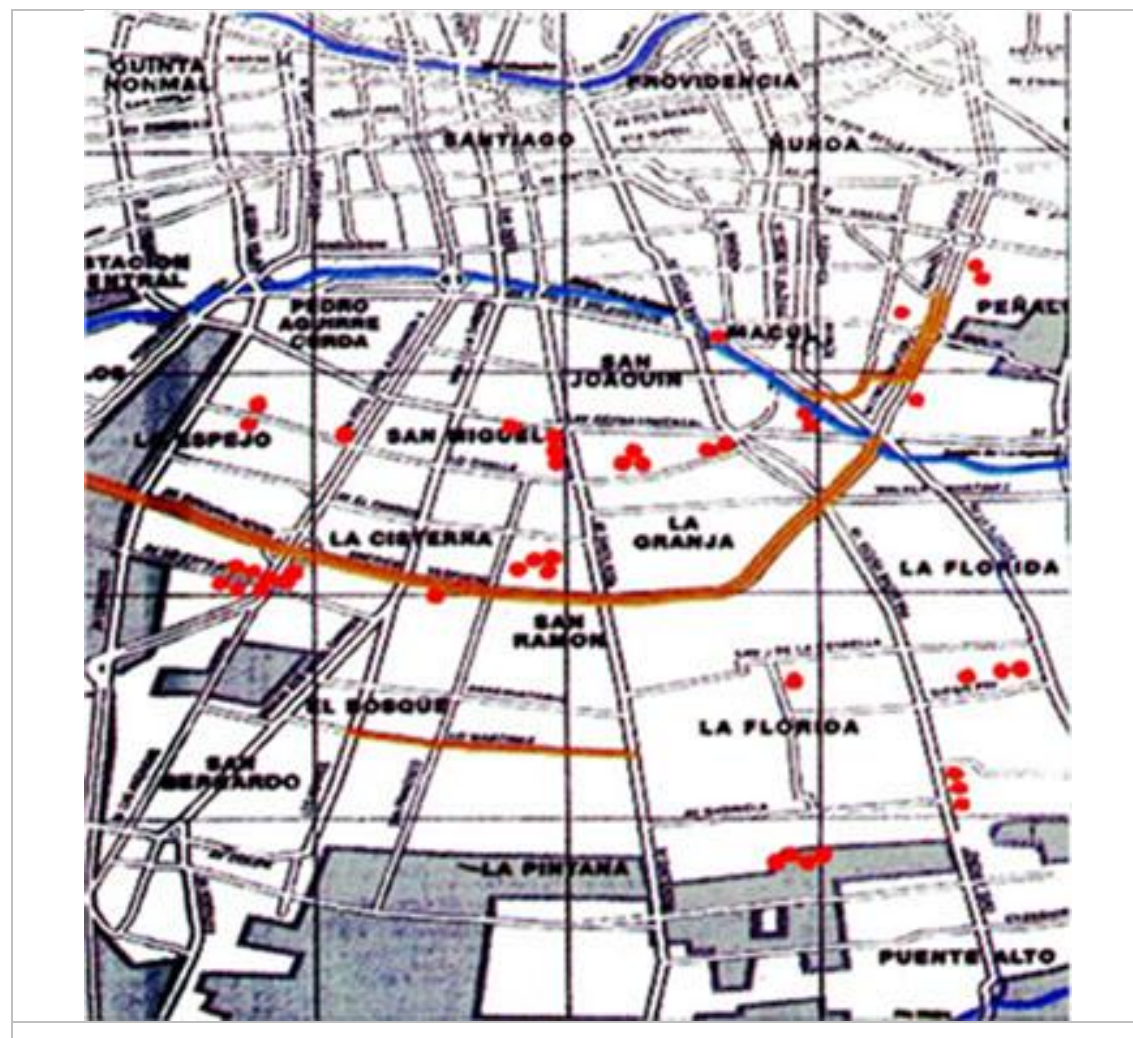

Fig.2.- Mapa que muestra los distintos lugares donde se han construido 44 pozos de infiltración para mitigar los efectos de las lluvias. 

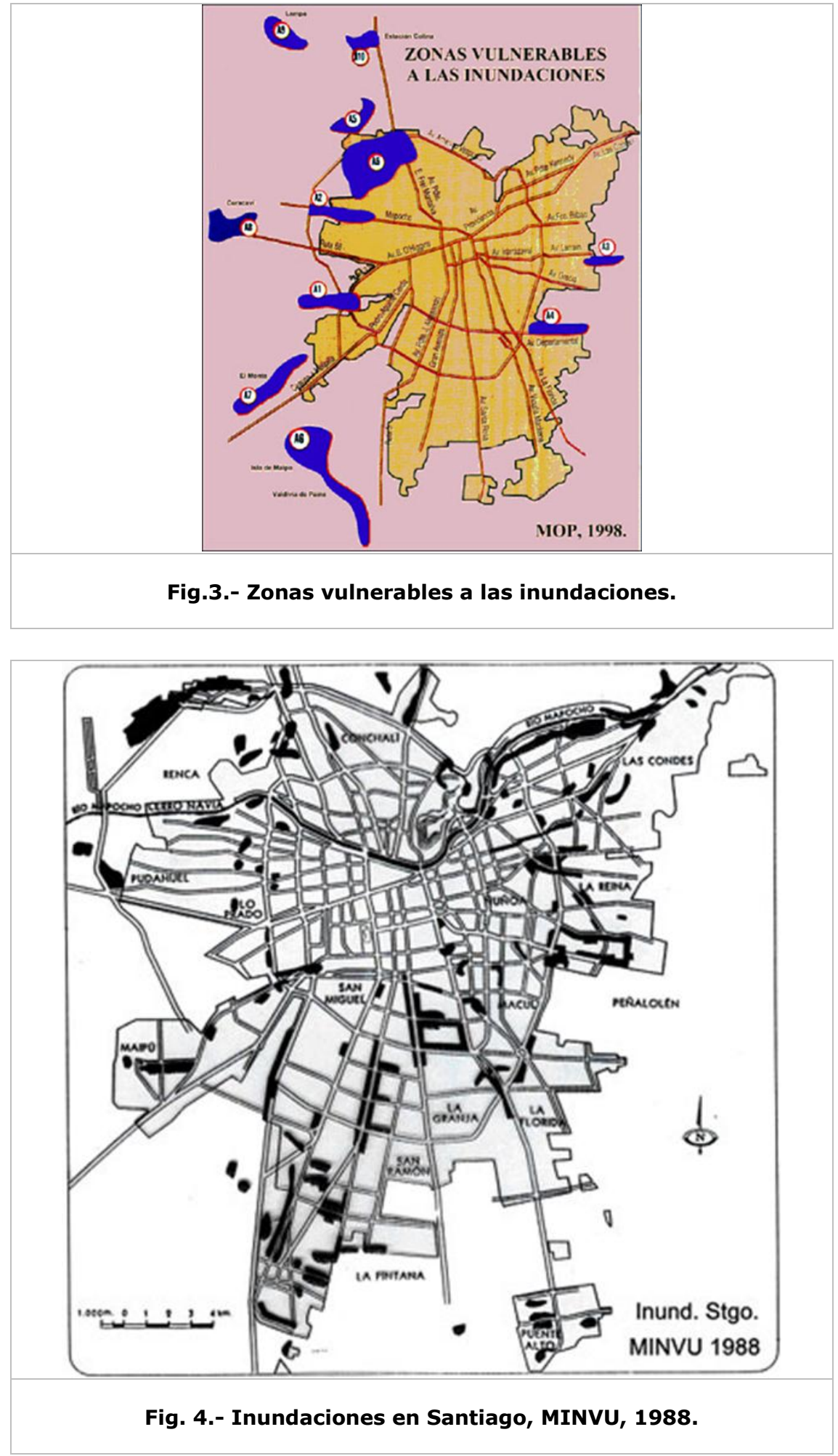


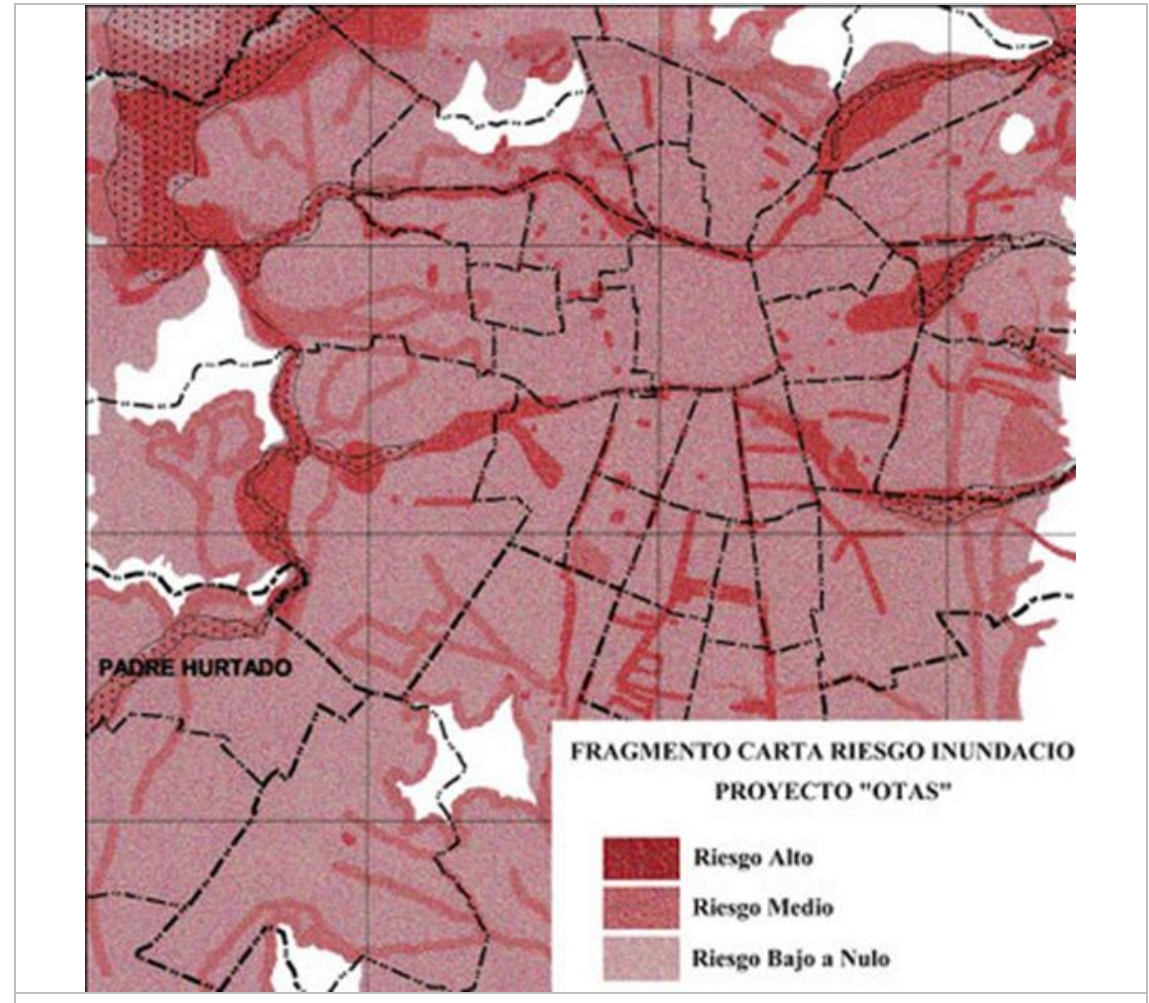

Fig. 5.- Fragmento Carta Riesgo Inundación. Proyecto OTAS.

Lógicamente, se requiere de muchos más pozos de infiltración, al menos en cantidad suficiente al $50 \%$ del volumen de las aguas que escurren por una ciudad dada en un invierno promedio. La ventaja de este tipo de obras es que son mucho menos invasivas y producen menos trastornos en la funcionalidad urbana que la construcción de colectores de aguas lluvias, últimos que implican levantar calles enteras. El uso de sistemas de pozos de infiltración de aguas lluvias es claramente ventajoso y factible en centros urbanos menores, $y$ se debería ir incrementando en número y complementando con colectores en el caso de ciudades intermedias y mayores según sea la situación local, última cuya determinación corresponde a lo que al respecto indican los antecedentes históricos de cada ciudad.

\section{Aportes del manejo de cuencas}

En el escenario en que el cambio climático global nos coloca, cualquiera sea la causa, la gestión integral de cuencas aparece como una herramienta que puede contribuir a la mitigación de los procesos de inundación, especialmente en aquella parte que deriva de las manifestaciones a las que la naturaleza nos está sometiendo.

Siendo las cuencas hidrográficas sistemas naturales que conforman unidades espaciales constitutivas de los territorios, del mismo modo que las células de un tejido orgánico, no hay lugar de las superficies continentales que no pertenezca a una de ellas. Visto así, estos sistemas naturales y dinámicos se convierten en unidades de gestión en los que el efecto mariposa está siempre presente. Lo que se haga en un 
lugar de una cuenca repercutirá, más tarde o más temprano, en el resto de ella. Las intervenciones modificantes de sus características naturales en un sentido negativo y, en respuesta, el manejo de cuencas en un sentido positivo, al buscar retrotraer o resarcir los efectos negativos mediante intervenciones adecuadas en tiempo y lugar.

Uno de los enfoques básicos es que los procesos que ocurren al interior de las cuencas, sean naturales o potenciados por acción humana, deben ser controlados desde su inicio, es decir, desde el nacimiento de los cursos de agua. Es muy difícil que tengan éxito medidas aplicadas en las secciones medias e inferiores de los sistemas de drenaje si la causa del evento se viene potenciando desde la sección superior de las cuencas. Sería como intentar frenar un tren desbocado con unos sacos de arena.

En este sentido, por ejemplo, es claro que mantener o aumentar la capacidad de retención hídrica en las secciones superiores de las cuencas, mediante la protección o recuperación de la cobertura vegetacional nativa u otra equivalente, es una de las medidas más efectivas para reducir la magnitud de las crecidas. Si se agrega la aplicación de técnicas para reducir la velocidad del flujo hídrico y, consecuentemente, para reducir su capacidad erosiva y de transporte de sedimentos, la mitigación de los efectos de un evento de esta naturaleza será mucho más efectiva y, a la vez, hará también más efectivas las medidas aplicadas en las secciones aguas abajo.

Estas y múltiples otras metodologías desarrolladas en el seno de la gestión integral de cuencas han probado en diversos países, como los del área andina por ejemplo, su efectividad $y$, con ello, el ahorro significativo de pérdidas sociales, económicas y ambientales.

\section{El tema de la alerta temprana}

Respecto de los procesos en cuestión, los sistemas de alerta temprana son también una de las herramientas de gestión de la prevención de mayor factibilidad y más alta eficacia. Existe concordancia en que "un sistema de Alerta de Crecidas resulta ser una herramienta de suma utilidad al momento de adoptar medidas de emergencia en tiempo real, ya que permite en algunos casos evitar y minimizar la pérdida de vidas humanas y bienes materiales" (González et als, 2001).

"Generalmente, un sistema de alerta está compuesto de un modelo matemático que permite pronosticar la variable de interés y de un modelo de decisiones que, alcanzado cierto umbral entrega una decisión de alertar o evacuar un cierto lugar" (González et als, op. cit.).

En este caso, la información requerida es la correspondiente base histórica de las crecidas ocurridas en un río dado que han provocado desbordes. Sin embargo, y existiendo modelos adecuados, este sistema de alerta de crecidas no está implementado en Chile, o bien, no funciona.

Cabe recordar que para el caso del Río Biobío, en el III Encuentro de las Aguas (IICA MOP, 2001), González et als (op. cit.) presentaron un Modelo Neuronal que permite establecer el nivel de alerta pertinente con 20 horas de antelación a una crecida de este río. Dicho modelo para la situación sinóptica de Julio-2006 claramente no fue utilizado. Lo más probable es que, de haberse hecho, se habrían salvado tantas vidas y 
bienes como los sistemas de emergencia actuando en forma coordinada hubieran podido. (Ver Figs. 6 y 7 ).

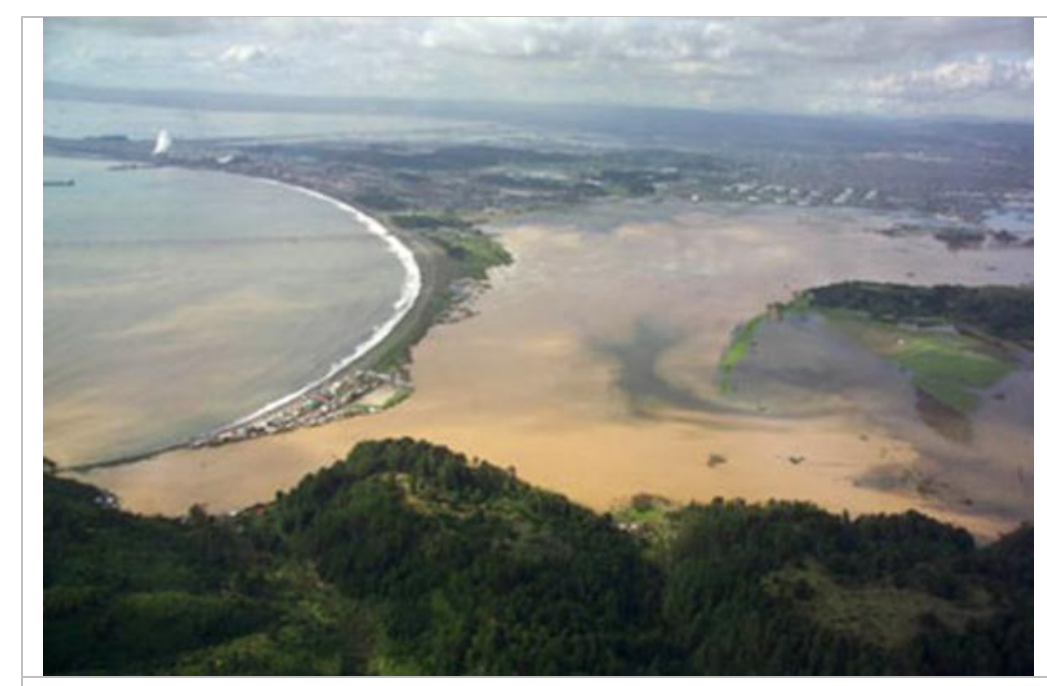

Fig.6. Inundación Julio 2006 sector Caleta Lenga, Río Biobío.

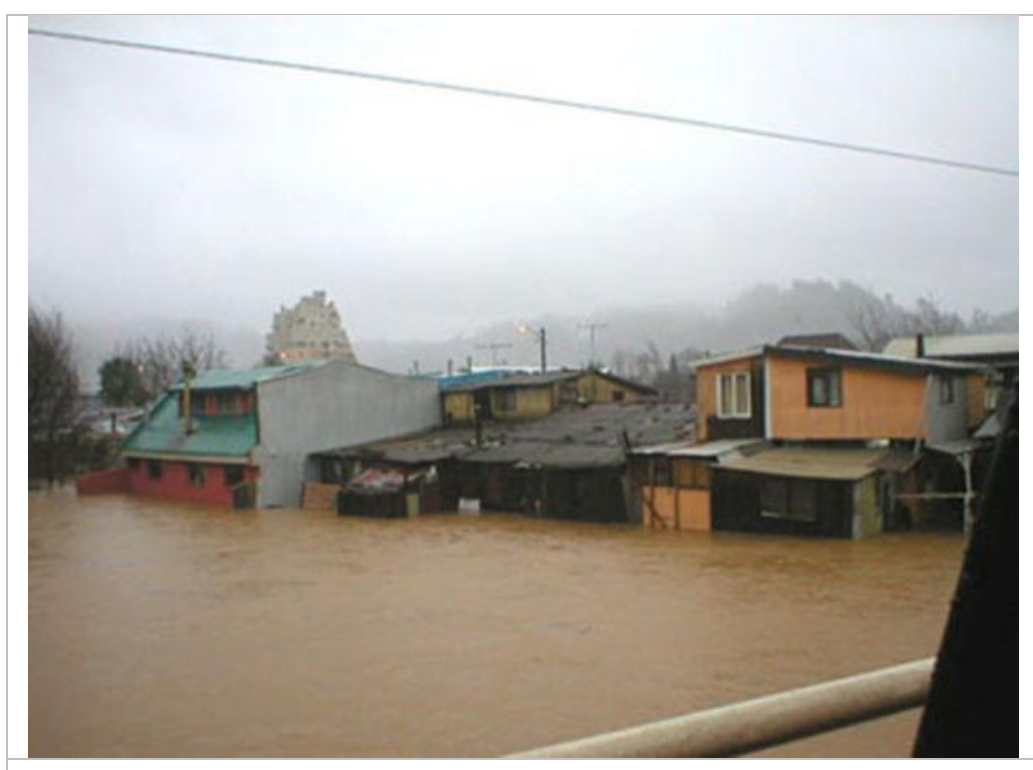

Fig.7. Inundación de casas en Concepción. Julio 2006.

Cabe pensar que dado el escenario pluviométrico y las experiencias de años anteriores, si la situación era previsible, por una parte no existió la decisión oportuna de alertar a la población y, por otra, alertadas las personas en forma oficial (i?) o por sus propias vivencias, se sacrificaron permaneciendo con sus pertenencias aun arriesgando la vida, producto de que nunca faltan los "amigos de lo ajeno", ese grupo infrahumano que es capaz incluso en tiempos de desastre de cometer ilícitos tan repudiables. 


\section{Sistematizando el quehacer sobre las inundaciones}

Sintetizando, este tipo de eventos se pueden desglosar como sigue:

Causas de las Inundaciones. Crecidas y desbordes de origen:

- Climático:

- Lluvias intensas

-Fusión intensa de la nieve acumulada

- Precipitaciones prolongadas

- Accidentales:

- Ruptura de represas

- Caída de frentes de hielo

- Deslizamientos de tierra

- Derrumbes

- Caída de árboles

- Caída de puentes

Hidromorfológico y/o antrópico:

- Desequilibrio entre el volumen en evacuación y la capacidad de colectores naturales y artificiales.

- Artificialización de los cauces con reducción de la sección por bajo el nivel de las altas aguas medias.

- Colmatación u obstrucción previa, natural o artificial, de cauces.

\section{Factores de las inundaciones}

\section{Factores Condicionantes:}

- Incremento considerable y momentáneo de la alimentación de una cuenca.

- Alta compacidad y pendiente en la zona de alimentación

- Baja pendiente en la zona de evacuación y sedimentación 
- Sección de cauces reducidos u obstruidos (colmatados)

\section{Factores Coadyuvantes:}

- Altas temperaturas primaverales (efecto: fusión nival violenta)

- Incremento de la impermeabilidad

- Angostamiento de cauces por obras de infraestructura

- Movimiento de tierras al interior del cauce

- Falta de limpieza y acondicionamiento de los cauces

\section{Procesos}

- Desborde de cauces

- Socavamiento y desmoronamiento de riberas

- Depositación de material estéril fuera del lecho

\section{Consecuencias}

- Arranque o botadura de árboles

- Daños a la infraestructura urbana

- Daños a la propiedad pública y privada

- Destrucción de cultivos

- Pérdida de fertilidad y arabilidad de suelos agrícolas

- Problemas de salud.

\section{Evaluación}

De acuerdo a lo señalado, la evaluación de los procesos de inundación, el peligro de ocurrencia, y sus consecuencias debiera considerar, en sus distintos ámbitos espaciotemporales, lo siguiente:

Evaluación de la Amenaza (proceso natural - o antrópicamente intervenido - que puede que generar la inundación)

Análisis de Antecedentes históricos:

- Situaciones sinópticas previas

- Productividad de la lluvia

- Áreas afectadas 
- Recurrencia de crecidas

- Magnitud de crecidas y desbordes

Determinación de Umbrales de Desencadenamiento en relación con:

- Montos pluviométricos

- Espesor de las cubiertas nivales

- Registros de temperaturas

Conducente a:

- Zonificar las áreas de producción de las amenazas.

- Diseñar e implementar medidas de control y/o mitigación en su fase genética de acuerdo al nivel y recurrencia de estas.

\section{Evaluación del Riesgo}

- Determinación espacial de la Exposición a la amenaza.

- Análisis de la estructura o diseño de las prevenciones tomadas e implementadas, si existen, y su funcionamiento en similares circunstancias.

- Confrontación de las Áreas amagadas versus el uso actual o planificado del suelo.

Conducente a llevar a cabo:

- Una Zonificación de Áreas en Riesgo por desbordes e Inundación.

- Una Planificación acorde del uso del suelo

- El diseño e implementación de Programas de Prevención que consideren medidas de:

- Elusión (erradicación)

- Mitigación

-Concepción y aplicación de medidas estructurales, biológicas y normativas.

\section{Evaluación de Consecuencias}

- Dimensionamiento de daños y pérdidas

- Cartografía de zonas afectadas según proceso y nivel de efecto

- Análisis de la efectividad de las medidas implementadas y obras ejecutadas. 
Conducente a:

- El perfeccionamiento de planes y medidas.

- La redefinición de la zonificación de áreas de riesgo, si fuese necesario.

- El reforzamiento y mantención de las obras.

\section{Conclusiones: tropezando con la misma piedra}

Sólo a modo de ejemplo, en el caso de la ciudad de Concepción, uno de los sistemas hidrológicos locales que históricamente ha sido el causante de la inundación de importantes sectores urbanos es la cuenca del Río Andalién.

Según Mardones et als (2006), las áreas con alto riesgo de inundación fluvial se han ido incrementando en el curso inferior del Río Andalién, así como del Estero Nonguén, como resultado del crecimiento y densificación de la ciudad en dicho sector. Se trata, ni más ni menos, que de la ocupación de las áreas de desahogo del río en momentos de crecidas. Qué se puede esperar entonces si paralelamente con ello no se han llevado a cabo las correspondientes medidas de manejo, no sólo del cauce en el sector, sino de la cuenca en su conjunto. Es evidente que ante cada invierno lluvioso el problema se repetirá.

Esta situación es común a muchos ríos de Chile, quizá demasiados. Esta realidad, tan asociada al determinismo hidrológico nacional es derivada, entre otros aspectos, tanto de la configuración de nuestro territorio como a la ocupación y explotación irresponsable de las cuencas, principalmente en términos de incremento del desequilibrio del sistema natural y de la torrencialidad de las respuestas hidrológicas.

Esto requiere, evidentemente, de una política de Estado y del destino de fondos para que, alguna vez, se dé inicio en forma concreta a programas de manejo de las cuencas, y no sólo de los cauces de las secciones medias y bajas de los ríos de montaña, acciones que hasta la fecha no han demostrado ser lo suficientemente eficientes para controlar los exabruptos hidrológicos propios de nuestros ríos o, mejor dicho, torrentes.

Si se sigue viendo el problema a escala de cauce y no a escala de cuenca, es claro que... "seguiremos tropezando con la misma piedra"... y lamentando las consecuencias directas e indirectas, cuyos costos tanto mal le hacen a los planes de desarrollo y a la calidad de vida de los chilenos.

\section{Bibliografía}

FERRANDO, F. 1994: "Métodos Hidromorfométricos para determinar la Erosividad a nivel de Cuencas Hidrográficas". Revista Ingeniería Hidráulica en México IX (3): 514. Septiembre Diciembre 1994. México. 
FERRANDO, F. 1995: "Clasificación Hidrodinámica de Chile". Cuadernos de Investigación Geográfica. Universidad de La Rioja, Logroño, España. Tomos XVIII / XIX (1992 1993): 57-74.

FERRANDO, F., CUETO S. 1998 Carta De Riesgos Naturales: Inundación. En: Proyecto Regional Bases Para El Ordenamiento Territorial Ambientalmente Sustentable De La Región Metropolitana De Santiago. Segunda Etapa: Inventario Ecológico y Evaluación del Territorio. Págs. 117-126.

FERRANDO, F. 2004. Alcances en Torno a la Gestión Ambiental a nivel de Cuencas Hidrográficas. Revista Tiempo y Espacio. Universidad del Biobío. Año 10-1993, No 13, págs. 175-184.

GONZÁLEZ ET ALS. 2001. Sistema de Alerta de Crecidas en el Río Biobío. Uso Recursivo de un Modelo de Redes Neuronales. III Encuentro de las Aguas IICA MOP. Santiago, Chile. 11 págs.

QUINTANILLA, V. 1990. Síntesis Cartográfica del Atlas del Ambiente de santiago Metropolitano: Estado de Avance. Revista Geográfica de Chile Terra Australis 32: 135-152.

\section{Otras fuentes}

ALCAÍNO, A. 1988. Estudio de las áreas de riesgo de inundación del Zanjón de la Aguada.

AYALA, L. H. 1990. Comportamiento hidráulico y mecánico fluvial de cauces cordilleranos y precordilleranos andinos: Caso del curso superior del río Mapocho.

AYALA, L. Y CABRERA, G. 1988a. Estudio de áreas de riesgo geofísico para asentimientos humanos.

AYALA, L. Y CABRERA, G. 1988b. Carta Hidrogeológica y Zonas de Inundación $1: 100.000$.

AYALA, L.; CABRERA, G. y Asociados Ltda. 1996. Análisis de la vulnerabilidad del sector oriente de la capital de Santiago ante la ocurrencia de aluviones y crecidas.

BOETTIGER, H. 1987. Evaluación del impacto de la inundación de julio de 1984 en la cuenca del estero Lampa.

COMISIÓN DEL PLAN INTEGRAL CONTRA INUNDACIONES. 1983. Recopilación de Estudios. Cuenca del río Mapocho.

COMITÉ REGIONAL DE EMERGENCIA. 1997. Propuesta medidas de contingencia para áreas de vulnerabilidad crítica. Intendencia Región Metropolitana.

INGENIERÍA INTEGRAL LTDA. 1992. Informe diagnóstico y priorización de 
proyectos aguas lluvias del gran Santiago.

KOVACIC S. I. 1991. Determinación de áreas de riesgos por inundación en la comuna de Vitacura. Revista Geográfica de Chile Terra Australis No 34.

LATORRE, P. 1986. Evaluación de impacto ambiental de las inundaciones de la comuna de San Bernardo.

MIDEPLAN - SGA. 1997. Estudio Piedmont y Cordillera Andina de Santiago. Etapa de diagnóstico. Escala 1:50.000.

MOP-CHILE 1994. Cartas de Áreas de Erosión e Inundación. Revisión Estudio de Factibilidad. Programa de "Manejo de Cuencas Hidrográficas". 1:50.000 (12 Hojas).

MOP-CHILE. 1994. Revisión Estudio de Factibilidad Programa Manejo de Cuencas Hidrográficas. Volumen I, punto 5.1.1.

MOP-CHILE. 1997. Consolidado de Emergencia en la Red Fluvial. R.M. Secretaría Regional Ministerial Metropolitana de Obras Públicas.

MOP-CHILE. 1997. Informe de Emergencia de Vialidad. R.M. Secretaría Regional Ministerial Metropolitana de Obras Públicas.

OLIVARES, M. S. 1987. Fenómeno de Inundaciones en la I. Comuna de Peñalolén.

ONU. DEPTO. DE ASUNTOS ECONÓMICOS Y SOCIALES. 1977. Directrices para la Prevención y Regulación de las Pérdidas debidas a las Inundaciones en los Países en desarrollo. Recursos Naturales/Serie del Agua No 5. ST/ESA/45.

SARAGONI, R. 1988. Áreas de Riesgo por Inundación Región Metropolitana 2a parte. Cuenca Maipo Mapocho.

URRUTIA Y LANZA. 1993. Antecedentes sobre temporales e inundaciones en la Región Metropolitana, entre 1957 y 1992. Memoria FCFM - Universidad de Chile. 